\title{
High-Resolution Sensors for the Operation and Control of a Novel Vacuum Evaporation Technology
}

\author{
Bence Fazekas, Krisztián Varga, Bálint Péter Szabó, Tamás Fráter and Gábor Simoncsics \\ PureAqua Environmental Engineering Company Ltd., Veszprém 8200, Hungary
}

\begin{abstract}
Industrial liquid waste streams could be significantly reduced by evaporation. Using evaporation technologies, not only the amounts are reduced, but the opportunities of potential economic reuse of the solutes are also promoted. In the process developed by authors' company, fast and efficient vaporization was achieved due to the high evaporation area and vacuum conditions. To investigate the details of the process, an experimental pilot equipment was constructed. The fine resolution monitoring of pressure and temperature conditions is essential in the system, which was implemented by a number of low response-time sensors. In this section of the research work, the developing and testing of the monitoring system customized for this aim was performed. Since the number of the sensors and the data rate is high, the control system was simplified (as possible), and instead of D/A converters author created a digital data management system. This system was capable of managing the 255 sensor's signals simultaneously in one channel, which seemed to be sufficient for this task. The error statistics of authors' system was excellent, even in industrial conditions.
\end{abstract}

Key words: Vacuum evaporation, monitoring sensors, data acquisition, bit error.

\section{Introduction}

Liquid waste streams, based on either water or organic solvents arise in numerous situations, in various branches of industry. The management of these wastes is a basic environmental and economical requirement nowadays. Since these solutions are usually dilute, the first step is a concentration, where a near pure solvent stream is recovered, and a concentrate is produced. Since the latter one is concentrated, its upgrading is more economic, including the cheaper and easier transport and the enhanced efficiency of separation technologies, such as crystallization, electrochemical technologies or evaporation [1-3]. This way, complete elimination of the liquid waste stream is possible (ZLD, Zero Liquid Discharge), which is nowadays a desirable aim in waste stream management [3, 4].

Membrane technologies are popular and prevalent methods for concentration, where the separation is

Corresponding author: Bence Fazekas, leader of R\&D Department, research field: wastewater treatment technologies. based on the molecular size (e.g. UF (Ultra Filtration), RO (Reverse Osmosis)), or the molecular affinity to the membrane (e.g. pervaporation, carrier-mediated technologies). All of them produce a larger amount (mostly 75-80\%) reusable solvent stream and a smaller amount (20-25\%) of concentrate. In wastewater treatment technologies, a general aim is the enhancement of the yield of the purified water, however, the concentrate remains a significant "problem", which sometimes has not been perfectly solved yet [5]. The solutes could be separated from this pre-concentrated stream more efficiently by electrochemical methods [2,5], crystallization [6], ion exchange, adsorption technologies [7] and evaporation [4]. These technologies are applied widely in waste stream management, and also could be integrated in industrial technologies such as food industry [8].

Among the aforementioned technologies for the upgrade of membrane concentrates, evaporation technologies are often applied. If the liquid volume is small, a simple atmospheric distillation is usually suitable, in case of larger amounts, the more economic 
vacuum evaporation is recommended [3]. However, vacuum evaporation is not adequate if the liquid contains foaming or gel-forming compounds such as oils, detergents etc. Among these, the non-ionic detergents are the most problematic components [9].

In authors' research work, an experimental vacuum evaporation system was built, where the vacuum was generated and sustained without using vacuum pumps. The dry matter solid content of the inlet liquid is crystallized as isolated crystals directly in the vacuum space. The special liquid distribution system allows the vaporization of "problematic" liquid waste streams, e.g. wastewaters containing foaming or gel-forming compounds are also suitable for this treatment. The evaporation is rapid, due to the large $\left(>1,000 \mathrm{~m}^{2} / \mathrm{L}\right)$ efficient evaporation and condensation surface.

The measurement and data registration during the evaporation is a great challenge because of the rapid changes in the physical-chemical parameters. The meet of the liquid inlet and the 20 mbar vacuum area in the system generates instant changes in the environmental parameters $[10,11]$, thus, the tracing of the phenomena requires rapid response sensors. The intense aerosol flow in the vaporization area excludes the use of non-contact measurement methods, however, the conventional contact temperature transmitters from the industrial applications are also not suitable for this aim, due to their minute-magnitude response time. High accuracy was also necessary. Moreover, significant mechanical and chemical protection is also necessary because of the rigid circumstances. The effect of $\mathrm{A} / \mathrm{D}$ converter had to be eliminated as well $[12,13]$.

The major development work was to find out the sensing/monitoring system which meets the aforementioned requirements.

\section{Material and Methods}

The PureAqua Environmental Engineering Company Ltd. in Veszprém, Hungary has been working on the development on vacuum evaporation technologies for many years. In the frame of the recent work, a vacuum evaporation system has been developed, especially for the one-step reduction of the amount of liquid waste streams and liquid hazardous wastes without volatile compound content. In the system, vacuum is generated by an alternative way (no pump is necessary), and the specific energy consumption is really favorable, thus the treatment of the aforementioned wastes became much more economic. Authors' aim is the significant reduction/elimination of the liquid waste discharges of the industrial technologies, moreover, the promotion of their reuse through concentration or crystallization.

\subsection{Experimental Set-Up}

The pilot plant is a 20 meter tall apparatus, containing near 1,000 components. Its central compartment is the evaporation tank (Fig. 1) for the inlet and evaporation of the feed (e.g. contaminated water). Salt crystals formed during the vaporization are collected in the inner surface of the evaporation tank. Then, the vapour phase is transported in the 9 meter tall condenser (Fig. 2), where it is cooled and the condensation is performed. The condensate is collected in the bottom and leads away by a tap. The waste heat generated by the condensation is transported and recycled in the pre-heating of the inlet, and partially used for the heating of the evaporation tank, optionally.

\subsection{Data Monitoring and Acquisition System}

In authors' experimental pilot plant, the crystallisation of the solid content is very rapid, usually, it happens in a few hundredth of a second! Thus, high resolution sensors are planned into the system, to get accurate and detailed information of the nature of evaporation and crystallization phenomena, and the flow parameters in the whole apparatus. Authors think that this information is very useful for the understanding of the aforementioned phenomena and thus, for the further development of industrial plants. However, it is also a 


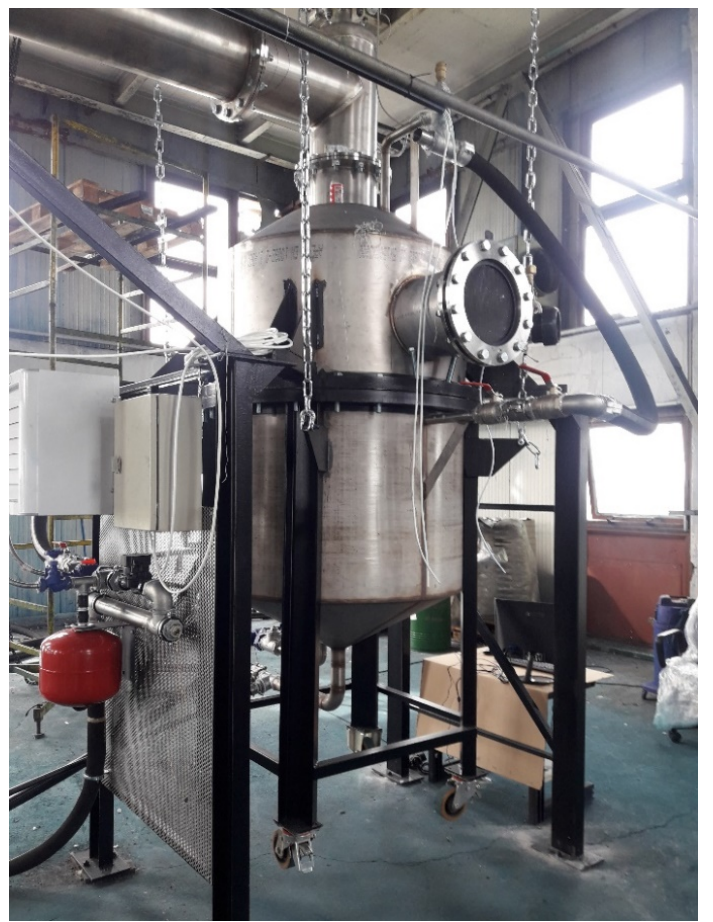

Fig. 1 Evaporation tank.

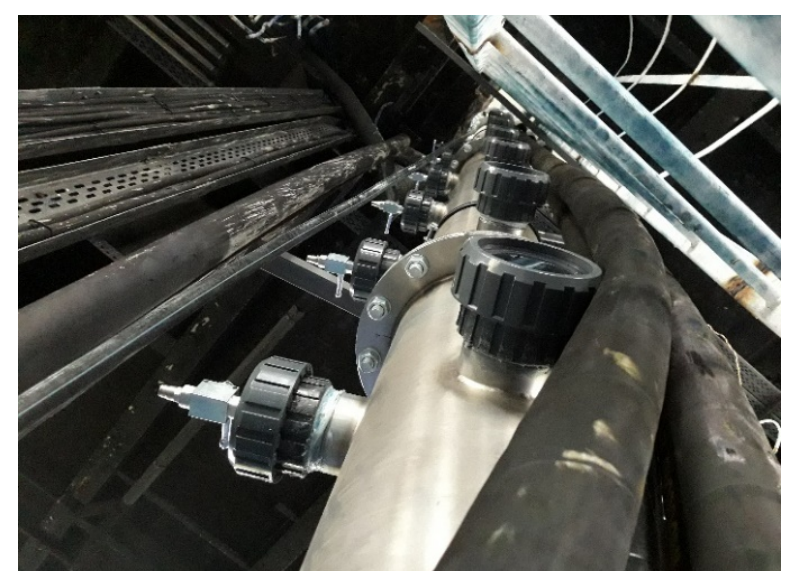

Fig. 2 Condenser.

great challenge in this development, since huge number of data from numerous parts of the apparatus with high resolution and high refreshing rate should be managed simultaneously.

During the vacuum evaporation, pressure conditions should be monitored with the accuracy of 0.1 millibars $(10 \mathrm{~Pa})$ and temperatures with the accuracy of $0.1{ }^{\circ} \mathrm{C}$. These parameters should be monitored in many points (altogether 50 points in the apparatus) with short response time (ca. $0.1 \mathrm{~s}$ ), however the architect of the monitoring system allows even lower (ca. $0.01 \mathrm{~s}$ ) response times, but it is focused only on a specified group of sensors. The big data number provides some advantage as well; the measurement inaccuracies could be partially eliminated by a continuous software filtering based on the statistic evaluation of the data mass. This way, the accuracy is enhanced by a software tool. Using these methods, really accurate data matrices are generated during the experiments, where the details of the processes could be monitored.

The sensors applied in the pilot plant possessed both digital and analogue outputs, and the communication network was a direct digital system, which was configurable without limits. To achieve this flexibility, no A/D converters were applied. Considering these, a relatively simple, fast-response, and also low-cost monitoring system was assembled.

Even this way, the pilot plant became, however, complicated, due to the huge number of sensing points for on-line monitoring of numerous parameters, e.g. temperature, pressure, flow rate. One part of the P\&ID drawing is presented in Fig. 3. These sensors were connected with the central data acquisition system by a digital communication network.

The most important advantage of the digital communication network is the fact, that 255 sensors could be integrated in only one spinal cord, by using various and unlimited numbers and types of junctions. The sensors possess individual identity labels, however, their physical status in the plant can be changed without any re-programming of the system.

Experiments were carried out with model solutions: $10 \%(\mathrm{~m} / \mathrm{m}) \mathrm{NaCl}$ (techn. grade, Sigma-Aldrich) in distilled water. In one batch, 22 liters solution was fed.

\section{Results}

During the first period, using model salt solutions, the system suitability was tested, and the basic parameters were set. The inlet was aqueous $\mathrm{NaCl}$ solution $(10 \%)$, and the product was almost salt-free condensate, and the salt forming in the evaporation was also visible. 


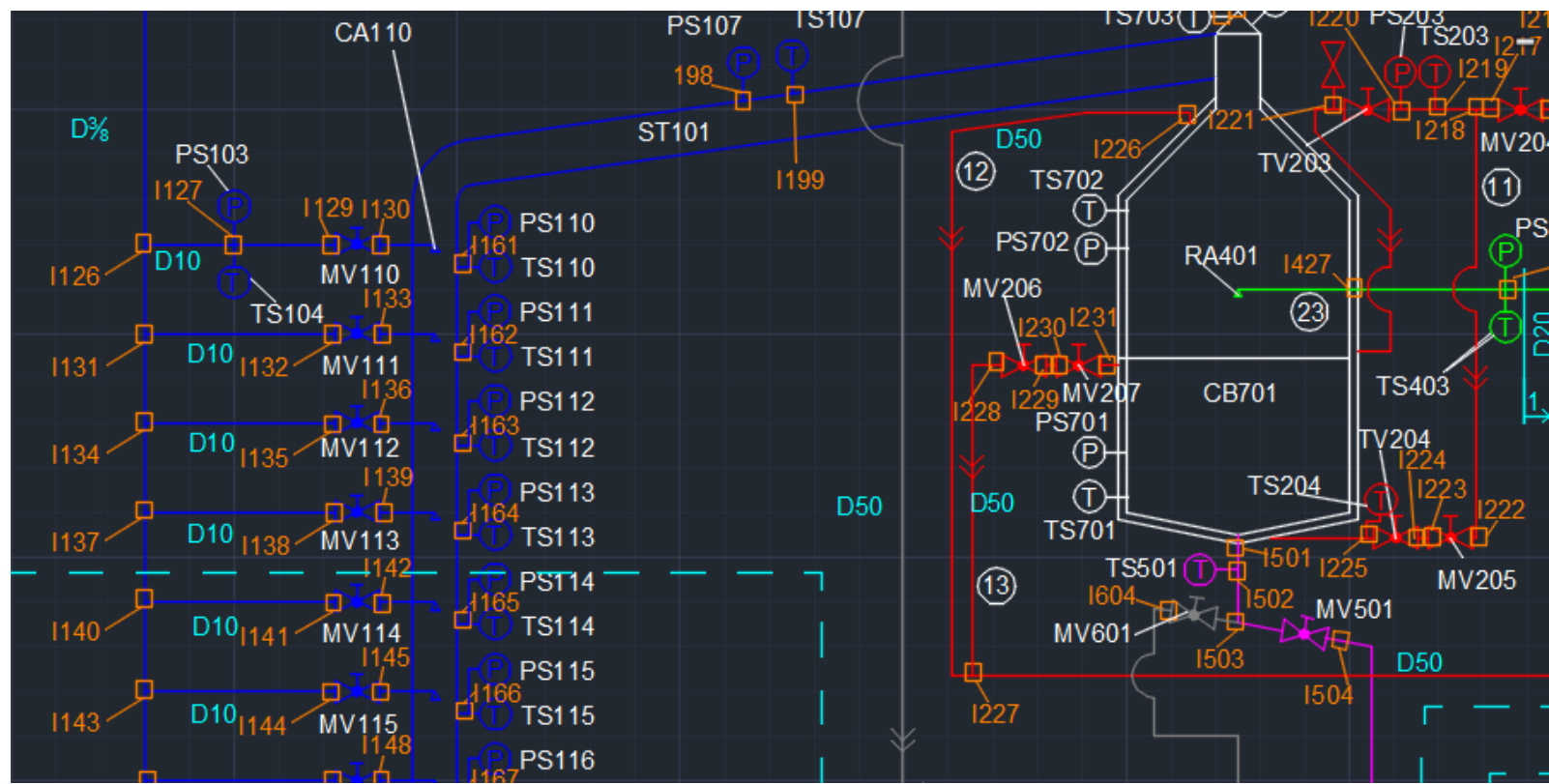

Fig. 3 A part of the P\&ID plan of the experimental pilot plant.

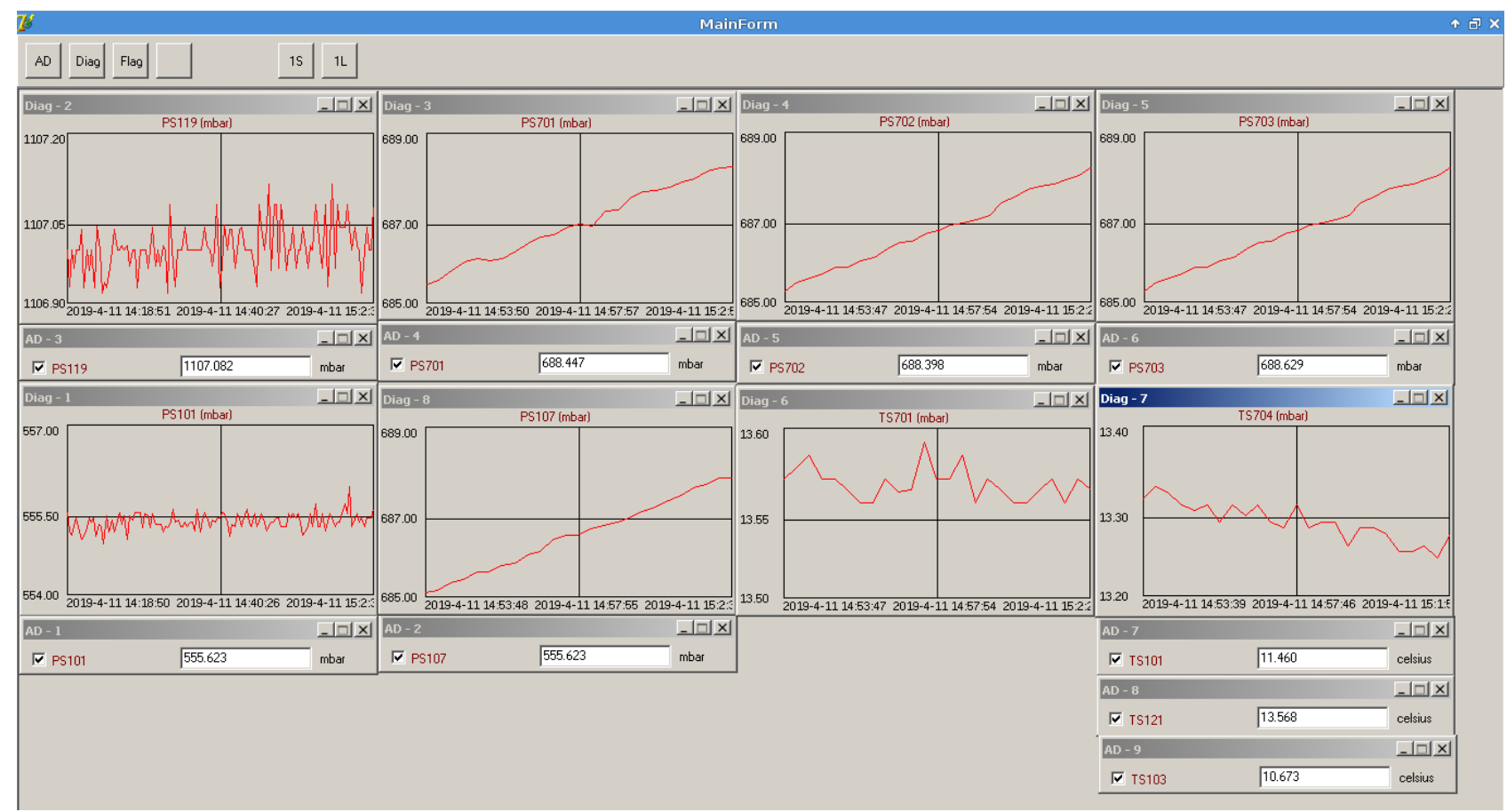

Fig. 4 Screenshot of the diagrams during the measurements.

The on-line parameters are visualized on the control panel (Fig. 4), which is configurable. During the tests, the control system, including the sensors, worked well, moreover, the error statistics of the data transfer was excellent.

The data traffic of sensing is 29-30 Mbytes daily. The communication on the network (control) means another and bigger part of the data traffic; this way 4-5-fold of the collected (sensor) data are transported through the system, in digital form. The whole amount of data transported in the first 50 days of the test period was about 55 gigabits. However, only one bit (!) was reported during this period by the automatic checking protocol as "communication bit error". 
Table 1 Bit errors (BER) compared to literature data.

\begin{tabular}{lll}
\hline Device & Reference & BER \\
\hline 8 bit converter & {$[12]$} & $6.2 \% 0$ \\
8 LSB converter (12 bit) & {$[14]$} & $1.7 \% 0$ \\
Bluetooth Medical Imaging & {$[15]$} & $\approx 10^{-4}$ \\
WLAN & {$[15]$} & $\approx 10^{-9}$ \\
UWB Serial Link Rx & {$[15]$} & $\approx 10^{-12}$ \\
Our apparatus & - & $1.8 \times 10^{-11}$ \\
\hline
\end{tabular}

In information technology, errors in communication are usually expressed as "BER" (Bit Error Rate). A conventional industrial data transport is operated with 1-3\% BER, mostly due to the electromagnetic noise in the industrial environment. Authors' research group has experience with a network operating with near $30 \%$ BER due to the radio communication. However, this system was still operable even with this really poor communication error rate! In the presented work, authors' BER is $1.83 \times 10^{-11}$, which is considered excellent in industrial environments, as compared to data from the literature (Table 1).

Authors note, that the only bit error in the test period occurred from an old technical element, the sensors developed for this project has not made any errors yet.

\section{Conclusions}

A system capable of the simple and efficient treatment of liquid wastes containing gel- or foam-forming agents was created in the frame of this project. The monitoring of all phenomena in the evaporation system was successfully achieved by a monitoring and data acquisition system developed by us. This system operates with excellent data error statistics compared to other systems. Authors' aim is to find out the optimal circumstances of the operation, and to develop an industrial size evaporator based on the observations.

\section{Acknowledgements}

The project was financed by the support of European Regional Development Fund in the frame of prototype development project, with the co-financing of the Hungarian Government (Project support ID: GINOP-2.1.7-15-2016-02233). The amount of the support was 45.2 million HUF.

\section{References}

[1] Cornwell, D. A., and Lee, R. G. 1994. "Waste Stream Recycling: Its Effect on Water Quality." Journal of American Water Works Association 86 (11): 50-63.

[2] Liu, C. K., and Li, C. W. 2004. "Simultaneous Recovery of Copper and Surfactant by an Electrolytic Process from Synthetic Solution Prepared to Simulate a Concentrate Waste Stream of a Micellar-Enhanced Ultrafiltration Process." Desalination 169: 185-92.

[3] Tong, T., and Elimelech, M. 2016. "The Global Rise of Zero Liquid Discharge for Wastewater Management: Drivers, Technologies, and Future Directions." Environmental Science and Technology 50 (13): 6846-55.

[4] Khan, S. J., Murchland, D., Rhodes, M., and Waite, T. D. 2009. "Management of Concentrated Waste Streams from High-Pressure Membrane Water Treatment Systems." Critical Reviews in Environmental Science and Technology 39: 367-415.

[5] Badruzzaman, M., Oppenheimer, J., Adham, S., and Kumar, M. 2009. "Innovative Beneficial Reuse of Reverse Osmosis Concentrate Using Bipolar Membrane Electrodialysis and Electrochlorination Processes." Journal of Membrane Science 326: 392-9.

[6] Randall, D. G., Nathoo, J., and Lewis, A. E. 2011. "A Case Study for Treating a Reverse Osmosis Brine Using Eutectic Freeze Crystallization-Approaching a Zero Waste Process." Desalination 266: 256-62.

[7] Kentish, S. E., and Stevens, G. W. 2001. "Innovations in Separations Technology for the Recycling and Re-use of Liquid Waste Streams." Chemical Engineering Journal 84: 149-59.

[8] Garcia-Castello, E., Lora-Garcia, J., Garcia-Garrido, J., and Rodriguez-Lopez, A. D. 2006. "Energetic Comparison for Leaching Waste Liquid from Citric Juice Production Using Both Reverse Osmosis and Multiple-effect Evaporation.” Desalination 191: 178-85.

[9] Gutiérrez, G., Benito, M., Coca, J., and Pazos, C. 2010. 
"Vacuum Evaporation of Surfactant Solutions and Oil-in-Water Emulsions." Chemical Engineering Journal 162: 201-7.

[10] Silveira, A. C. P. 2015: "Thermodynamic and Hydrodynamic Characterization of the Vacuum Evaporation Process during Concentration of Dairy Products in a Falling Film Evaporator. Food and Nutrition." https://tel.archives-ouvertes.fr/tel-01342521/document.

[11] Shahzad, M. W., Burhan, M., and Ng, K. C. 2017. "Development of Falling Film Heat Transfer Coefficient for Industrial Chemical Processes Evaporator Design." In Statistical Approaches with Emphasis on Design of Experiments Applied to Chemical Processes, Intech Open, Chapter 8.
[12] Azman, M., Latif, A., Basheer, N., Zain, A., and Hussin, F. A. 2011. "NBTI-Induced 8-Bit DAC Circuit Mismatch in System-on-Chip (SoC)." Presented at 3rd Asia Symposium on Quality Electronic Design, 19-20 July, Kuala Lumpur, Malaysia.

[13] Michaeli, L., and Šaliga, J. 2014. "Error Models of the Analog to Digital Converters." Measurement Science Review 14 (2): 62-6.

[14] Baker, B. 2004. "Number of Bits versus LSB Errors." https://www.edn.com/electronics-blogs/bakers-best/4330 029/Number-of-bits-versus-LSB-errors.

[15] Dwobeng, E. 2013. "Measuring Bit Errors in the Output Word of an A/D Converter." SLAA582-April, CTexas Instruments Inc.. 